\title{
APOBEC3A/B-induced mutagenesis is responsible for $20 \%$ of heritable mutations in the TpCpW context
}

\author{
Vladimir B. Seplyarskiy, ${ }^{1,2}$ Maria A. Andrianova, ${ }^{1,2,3}$ and Georgii A. Bazykin ${ }^{1,2,3,4}$ \\ ${ }^{1}$ Institute for Information Transmission Problems of the Russian Academy of Sciences (Kharkevich Institute), Moscow 127994, Russia; \\ ${ }^{2}$ Pirogov Russian National Research Medical University, Moscow 117997 , Russia; ${ }^{3}$ Lomonosov Moscow State University, Moscow \\ 119234, Russia; ${ }^{4}$ Skolkovo Institute of Science and Technology, Skolkovo 143026, Russia
}

\begin{abstract}
APOBEC3A/B cytidine deaminase is responsible for the majority of cancerous mutations in a large fraction of cancer samples. However, its role in heritable mutagenesis remains very poorly understood. Recent studies have demonstrated that both in yeast and in human cancerous cells, most APOBEC3A/B-induced mutations occur on the lagging strand during replication and on the nontemplate strand of transcribed regions. Here, we use data on rare human polymorphisms, interspecies divergence, and de novo mutations to study germline mutagenesis and to analyze mutations at nucleotide contexts prone to attack by APOBEC3A/B. We show that such mutations occur preferentially on the lagging strand and on nontemplate strands of transcribed regions. Moreover, we demonstrate that APOBEC3A/B-like mutations tend to produce strandcoordinated clusters, which are also biased toward the lagging strand. Finally, we show that the mutation rate is increased $3^{\prime}$ of $\mathrm{C} \rightarrow \mathrm{G}$ mutations to a greater extent than $3^{\prime}$ of $\mathrm{C} \rightarrow \mathrm{T}$ mutations, suggesting pervasive trans-lesion bypass of the APOBEC3A/B-induced damage. Our study demonstrates that $20 \%$ of $C \rightarrow T$ and $C \rightarrow G$ mutations in the TpCpW context-where $\mathrm{W}$ denotes $\mathrm{A}$ or $\mathrm{T}$, segregating as polymorphisms in human population—or $1.4 \%$ of all heritable mutations are attributable to APOBEC3A/B activity.
\end{abstract}

[Supplemental material is available for this article.]

Understanding the processes responsible for heritable mutations is important for a broad range of evolutionary, population genetics, and medical questions (Shendure and Akey 2015). Recent studies have shown that different numbers of de novo mutations are inherited from the father and from the mother, with the contribution of paternal mutations being two to four times higher (Kong et al. 2012; Francioli et al. 2015; Wong et al. 2016; Yuen et al. 2016). Additionally, the number of de novo mutations strongly depends on the father's age at conception (Kong et al. 2012; Francioli et al. 2015; Wong et al. 2016; Yuen et al. 2016) and, to a lesser extent, on the mother's age at conception (Goldmann et al. 2016; Wong et al. 2016). At the molecular level, the understanding of mechanisms of heritable mutagenesis is very limited. Only a few of the mutation types can be attributed to specific molecular processes. Most prominently, the $\mathrm{CpG} \rightarrow \mathrm{TpG}$ substitutions are known to result from spontaneous cytosine deamination of methyl-cytosine in the $\mathrm{CpG}$ context together with poor efficiency of subsequent base excision repair (Pfeifer 2006; Chen et al. 2014); some mutations in the $\mathrm{CpCpC}$ motif arise due to activity of the APOBEC3G protein, which is normally involved in protection against viruses and retroelements (Knisbacher and Levanon 2016; Pinto et al. 2016), and small insertions and deletions result from polymerase slippage on homonucleotide tracts and tandem repeats (Montgomery et al. 2013).

The sources of somatic mutations, in particular those in cancers, are better understood. The rates of such mutations were related to age-dependent cytosine deamination, to activity of APOBEC3A/B/G and AID, to deficiencies in systems responsible for the fidelity of DNA repair and replication, and to exposure to external and internal mutagens (Alexandrov et al. 2013; Lawrence et al. 2013).

Corresponding author: alicodendrochit@gmail.com

Article published online before print. Article, supplemental material, and publication date are at http://www.genome.org/cgi/doi/10.1101/gr.210336.116.
APOBEC3A/B-induced mutations were described for many cancer types (Alexandrov et al. 2013; Burns et al. 2013b; Roberts et al. 2013). Mutations produced by APOBEC3A/B have known properties confirmed both in yeast and in human cancers: (1) They are $\mathrm{C} \rightarrow \mathrm{D}$ mutations in the Tp$\underline{\mathrm{C}} \mathrm{pN}$ context (the more specific APOBEC3A/B signature is TpCpW $\rightarrow K$, where D denotes A, T, or G; W denotes A or T; and K denotes G or T) (Burns et al. 2013a; Taylor et al. 2013; Chan et al. 2015; Seplyarskiy et al. 2016); (2) they often form strand-coordinated clusters (Nik-Zainal et al. 2012; Roberts et al. 2013; Taylor et al. 2013); (3) they are strongly biased toward the lagging strand during replication (Haradhvala et al. 2016; Hoopes et al. 2016; Morganella et al. 2016; Nik-Zainal et al. 2016; Seplyarskiy et al. 2016); (4) they are biased toward the nontranscribed strand, at least in breast and bladder cancer (Nordentoft et al. 2014; Morganella et al. 2016); and (5) cytosines deaminated to uracils by APOBEC frequently result in $\mathrm{C} \rightarrow \mathrm{G}$ substitutions. According to the current models, these mutations arise due to incomplete repair of U-G mismatches resulting in abasic sites. In turn, abasic sites are bypassed by REV1, which inserts exactly 1 nucleotide (nt) opposite to the site, and this single-nucleotide primer is then extended by the low-fidelity polymerase $\zeta$ (NikZainal et al. 2012; Chan et al. 2013; Seplyarskiy et al. 2015). Additionally, as we show here, (6) strand-coordinated clusters in cancers are very strongly biased toward the lagging strand.

We previously demonstrated that fork polarity (fp) calculated as the derivative of replication timing allows to discriminate between the strands replicated as leading versus lagging (Seplyarskiy et al. 2016) and that accumulation of APOBEC3A/B

(c) 2017 Seplyarskiy et al. This article is distributed exclusively by Cold Spring Harbor Laboratory Press for the first six months after the full-issue publication date (see http://genome.cshlp.org/site/misc/terms.xhtml). After six months, it is available under a Creative Commons License (Attribution-NonCommercial 4.0 International), as described at http://creativecommons.org/licenses/ by-nc/4.0/. 
mutations in human cancers is strongly strand specific. Here, we asked if this specificity, as well as other properties of APOBEC3A/ $\mathrm{B}$-induced mutagenesis, is also manifested in heritable mutations.

\section{Results}

Heritable mutations in the APOBEC3A/B context are $20 \%$ more frequent on the lagging strand

Rare polymorphisms tend to be young (Mathieson and McVean 2014). Therefore, their mutational spectra and genomic distribution much better reflect the spectra of de novo mutations compared with the spectra and distribution of substitutions between species that are affected by nonmutational processes such as selection or biased gene conversion operating over the lifetime of a mutation as it spreads to fixation (Rahbari et al. 2016; Terekhanova et al. 2016). To study heritable mutations, we thus mainly focused on rare polymorphisms (The 1000 Genomes Project Consortium $2015)$, i.e., those with the frequency of the derived allele in the human population $<1 \%$; we excluded singletons from the main analyses as they are enriched in cell line artefacts (Mathieson and Reich 2016).

We measured the ratio of the frequencies of $\mathrm{C} \rightarrow \mathrm{K}$ mutations to those of complementary $\mathrm{G} \rightarrow \mathrm{M}$ (where $\mathrm{M}$ denotes $\mathrm{A}$ or $\mathrm{C}$ ) mutations in different contexts and asked how this ratio depends on whether the analyzed strand is preferentially replicated as leading or lagging. The rates of both provisionally APOBEC3A/B-induced mutation types (to $\mathrm{T}$ and to $\mathrm{G}$ ) in both APOBEC $3 \mathrm{~A} / \mathrm{B}$ contexts (TpCpT and TpCpA) were 10\%-20\% higher on the lagging strand (Fig. 1), while a weak bias was observed in only one of the comparisons for the corresponding mutations in the non-APOBEC3A/B VpCpW context (where V denotes A, C, or G) (Fig. 1A). Among the 24 possible mutations in $\mathrm{Np} \underline{\mathrm{CpW}}$ contexts, the TpCpW $\rightarrow \mathrm{K}$ mutations are the most asymmetric. These estimates hold for all seven cell types with measured replication timing (Table 1; Supplemental Table S1).

The context specificity of the asymmetry, together with the known lagging strand preferences of APOBEC3A/B-induced mutations, suggests that the observed bias is caused by an excess of
APOBEC3A/B-induced mutations on the lagging strand. If so, we can estimate the contribution of APOBEC3A/B to mutagenesis, assuming that it is the sole reason for these biases (for details, see Methods). Under these assumptions, we infer that $15 \%-30 \%$ of $\mathrm{Tp} \underline{\mathrm{C}} \mathrm{W} \rightarrow \mathrm{K}$ substitutions resulted from APOBEC $3 \mathrm{~A} / \mathrm{B}$ deamination, with a lower fraction for the TpCpA $\rightarrow \mathrm{G}$ mutations $(\sim 15 \%)$. The differences in strand bias between different APOBEC3A/B con-

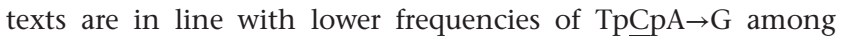
APOBEC3A/B-induced mutations in cancers (Alexandrov et al. 2013) and among APOBEC3B-induced mutations in human cells lines (Akre et al. 2016). Similar biases were observed for SNPs at all frequencies (Supplemental Table S2) and for interspecies differences accumulated in the human lineage since divergence from the last common ancestor with the chimpanzee, albeit they are slightly less obvious for divergence (Supplemental Table S2; Supplemental Fig. S1). Possibly, slightly weaker asymmetry observed in divergence reflect minor changes in RT, as it was shown that RT varies even within the human population (Koren et al. 2014).

APOBEC3A- and APOBEC3B-induced mutations preferentially occur when, respectively, Y (T or C) or R (A or G) is observed $2 \mathrm{nt}$ upstream of the mutated C (Taylor et al. 2013; Chan et al. 2015). Therefore, by analyzing this extended context, we are able to discriminate between these two enzymes. The strand bias is stronger for YpTpCpW contexts than for RpTpCpW contexts $(P<0.0024$ for all comparisons) (Supplemental Fig. S2), suggesting that similarly to cancers with a high burden of APOBEC-induced mutations (Chan et al. 2015), APOBEC3A likely contributes more to the observed mutations than APOBEC3B.

In cancers most affected by APOBEC3A/B-induced mutagenesis, the mutation rate in the TpCpW context is increased by up to 40-fold compared with the VpCpW context (Seplyarskiy et al. 2016). From strand asymmetry, we estimate that only 15\%-30\% of heritable $\mathrm{TpCpW} \rightarrow \mathrm{K}$ mutations are induced by APOBEC3A/B. We asked whether this APOBEC3A/B-induced mutagenesis is also manifested in an increased genome-wide mutation rate in the corresponding nucleotide context. However, the frequencies of $\mathrm{TpCpW} \rightarrow \mathrm{K}$ were not uniformly higher than the frequencies of $\mathrm{VpCpW} \rightarrow \mathrm{K}$ mutations (Supplemental Table S3). This is probably because mechanisms other than those associated with APOBEC3A/B, with their own context specificities (Supplemental Fig. S3; Aggarwala and Voight 2016), contribute more to polymorphism data compared with cancer samples where APOBEC3A/B virtually monopolizes the mutation process.

Heritable mutations in the APOBEC3A/ $B$ context are more frequent on the nontemplate strands of transcribed regions

To understand other genomic features that could be associated with APOBEC3A/B activity, we analyzed the density of rare polymorphisms in the APOBEC3A/B context in genomic regions that may be prone to deamination by APOBEC $3 \mathrm{~A} / \mathrm{B}$ : transcribed regions, recombination hotspots, and expressed transposons. No APOBEC3A/B-specific

\section{Genome Research}

www.genome.org 
APOBEC3A / B major source of heritable mutations

Table 1. APOBEC3A/B-driven replication asymmetry is concordantly observed in seven cell types with known RT

\begin{tabular}{|c|c|c|c|c|c|c|c|c|c|c|c|c|c|c|c|c|}
\hline & \multicolumn{2}{|c|}{ MCF-7 } & \multicolumn{2}{|c|}{ Hepg2 } & \multicolumn{2}{|c|}{ Imr90 } & \multicolumn{2}{|c|}{ K562 } & \multicolumn{2}{|c|}{ Gm12878 } & \multicolumn{2}{|c|}{ Nhek } & \multicolumn{2}{|c|}{ Sknsh } & \multicolumn{2}{|c|}{$\begin{array}{l}\text { MCF-7 } \\
\text { shuffled }\end{array}$} \\
\hline & Rank & $\log _{2}$ & Rank & $\log _{2}$ & Rank & $\log _{2}$ & Rank & $\log _{2}$ & Rank & $\log _{2}$ & Rank & $\log _{2}$ & Rank & $\log _{2}$ & Rank & $\log _{2}$ \\
\hline$T p C p T>T$ & 1 & 0.26 & 1 & 0.28 & 1 & 0.21 & 1 & 0.21 & 1 & 0.24 & 2 & 0.20 & 1 & 0.25 & 8 & -0.02 \\
\hline$T p \overrightarrow{C p T}>C$ & 2 & 0.24 & 2 & 0.24 & 2 & 0.19 & 2 & 0.19 & 2 & 0.24 & 1 & 0.22 & 3 & 0.15 & 5 & -0.03 \\
\hline$T p C p A>T$ & 3 & 0.23 & 3 & 0.24 & 3 & 0.17 & 3 & 0.19 & 3 & 0.21 & 3 & 0.20 & 2 & 0.18 & 13 & -0.01 \\
\hline$A p C p T>G$ & 4 & 0.18 & 4 & 0.19 & 6 & 0.15 & 4 & 0.17 & 4 & 0.18 & 4 & 0.18 & 5 & 0.14 & 7 & -0.02 \\
\hline$G p C p T>G$ & 5 & 0.16 & 8 & 0.14 & 5 & 0.15 & 5 & 0.16 & 6 & 0.15 & 6 & 0.15 & 10 & 0.10 & 12 & -0.01 \\
\hline$C p C p A>T$ & 6 & 0.15 & 5 & 0.17 & 7 & 0.13 & 8 & 0.12 & 7 & 0.14 & 5 & 0.18 & 6 & 0.11 & 21 & 0.00 \\
\hline$T p C p T>A$ & 7 & -0.15 & 13 & -0.12 & 9 & -0.11 & 7 & -0.13 & 13 & -0.10 & 8 & -0.13 & 7 & -0.11 & 4 & 0.03 \\
\hline$T p С p A>C$ & 8 & 0.15 & 7 & 0.16 & 4 & 0.16 & 6 & 0.14 & 5 & 0.16 & 10 & 0.13 & 4 & 0.15 & 3 & 0.04 \\
\hline$C p C p T>G$ & 9 & 0.14 & 10 & 0.12 & 8 & 0.11 & 11 & 0.09 & 9 & 0.13 & 7 & 0.14 & 9 & 0.10 & 9 & 0.01 \\
\hline$G p C p T>T$ & 10 & -0.13 & 6 & -0.16 & 10 & -0.11 & 9 & -0.12 & 8 & -0.13 & 9 & -0.13 & 11 & -0.09 & 22 & 0.00 \\
\hline
\end{tabular}

We list the top 10 mutation types in the NpCpW context with the highest asymmetry in MCF-7 cells. For each cell type, we show the rank of each mutation based on the absolute value of $\log _{2}$ of the ratio of mutation rates on the lagging and leading strands. TpCpW $\rightarrow \mathrm{K}$ mutations are in bold. "MCF-7 shuffled" is the asymmetry calculated for spurious RT values obtained by reshuffling the RT values between windows for MCF-7 data.

differences from the background rate were observed in recombination hotspots or in transposons (Supplemental Fig. S4).

For transcribed regions, we saw evidence for APOBEC3A/B activity at the nontemplate strand. Here, the rate of $\mathrm{TpCpW} \rightarrow \mathrm{K}$ mutations was increased compared with intergenic regions (Fig. 2A). No such increase was observed for the template strand (Fig. 2B). As a result, TpCpW $\rightarrow \mathrm{K}$ mutations were strongly biased toward the nontemplate strand. Indeed, at the nontemplate strand, the $\mathrm{TpCpT} \rightarrow \mathrm{G}$ mutations had the strongest mutational asymmetry among all 24 mutations in the NpCpW contexts, and other $\mathrm{Tp} \underline{\mathrm{p} W} \rightarrow \mathrm{K}$ mutations were also biased toward the nontranscribed strand (Fig. 2C). These observations are in line with the transcriptional asymmetry of APOBEC3A/B-induced mutations in bladder cancer and breast cancer (Nordentoft et al. 2014; Morganella et al.2016) and likely reflect the accessibility of the nontranscribed strand to APOBEC3A/B, because the nontemplate strand is prone to single-strandedness (Skourti-Stathaki and Proudfoot 2014). The replication asymmetry of $\mathrm{Tp} \underline{\mathrm{C}} \mathrm{W} \rightarrow \mathrm{K}$ mutations is observed both in transcribed and nontranscribed regions, implying that it is independent of the effect of transcription, although the extent of this asymmetry differs slightly between the template and the nontemplate strands because of contribution of chain-specific mutations (Fig. 2; Supplemental Table S4).

\section{Heritable mutations in APOBEC3A/B context tend to form strand-coordinated clusters}

As APOBEC family enzymes deaminate single-stranded DNA, they tend to produce strand-coordinated clusters. To study such clusters, we focused on pairs of rare single-nucleotide polymorphisms (SNPs) in strong linkage at distances of up to $5000 \mathrm{nt}$ from each other (for details, see Methods). Mutations at sites closer than $10 \mathrm{nt}$ to each other occur due to activity of low fidelity polymerases or other mechanisms not related to APOBEC3A/B (Terekhanova et al. 2013; Harris and Nielsen 2014; Zhu et al. 2015); therefore, we excluded such pairs from our analysis. Over half of the remaining pairs of $\mathrm{Tp} \mathrm{CpW} \rightarrow \mathrm{K}$ mutations were strand coordinated, and for three out of the four considered mutation types, the fraction of strand-coordinated pairs was significantly higher than for the same mutations in the VpCpW context used as a control (Fig. 3A-D). Similar results
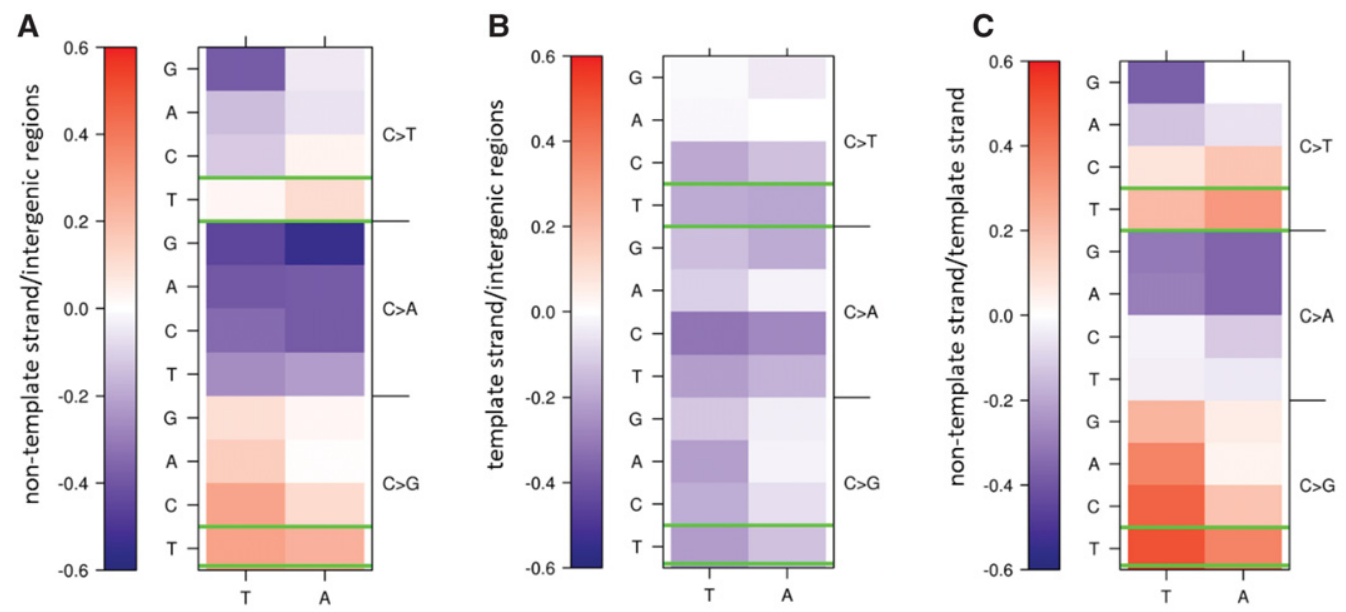

Figure 2. $A P O B E C 3 A / B$-induced mutations occur preferentially on the nontemplate strand. Color-coded $\log _{2}$ of the ratio of densities of rare polymorphisms on the nontemplate strand $(A)$ or the template strand $(B)$ and intergenic regions, or of the ratio of densities of rare polymorphisms on the nontemplate and template strands $(C)$. Mutations in the $\mathrm{Tp} \underline{\mathrm{C}} \mathrm{pW} \rightarrow \mathrm{K}$ context that may be associated with APOBEC $3 \mathrm{~A} / \mathrm{B}$ activity are in green boxes. 
were obtained for interspecies divergence data (Supplemental Table S5). Conversely, strand-coordinated clusters composed of many (more than six) $\mathrm{C} \rightarrow \mathrm{K}$ mutations were enriched in mutations in an APOBEC3A/B-prone context. Indeed, in such clusters, the fraction of $\mathrm{TpCpW} \rightarrow \mathrm{K}$ mutations was about three times higher than in noncoordinated clusters with similar properties (Fig. 3E). Moreover, many of the strand-coordinated clusters included only $\mathrm{TpCpN} \rightarrow \mathrm{K}$ mutations (Supplemental Table S6) and likely represented pure traces of APOBEC3A/B activity.

APOBEC3A/B-induced mutations are biased toward the lagging strand in cancer and in yeasts. We asked whether this pattern is also observed for strand-coordinated clusters. In cancers with a strong prevalence of APOBEC3A/B-related mutagenesis, strandcoordinated clusters occur sixfold more frequently on the lagging strand, demonstrating the highest level of replicative strand asymmetry reported for mammalian cells (Supplemental Fig. S5). Therefore, we would expect an excess of strand-coordinated pairs of heritable $\mathrm{TpCpW} \rightarrow \mathrm{K}$ mutations on the lagging strand if APOBEC3A/B plays a role in their formation. Indeed, clustered mutations preferentially occur on the lagging strand (Fig. 3F-I), and the level of strand asymmetry for them is higher than that for dispersed mutations (Supplemental Fig. S6), reflecting an enrichment of APOBEC3A/B-induced clusters among strand-coordinated clusters.
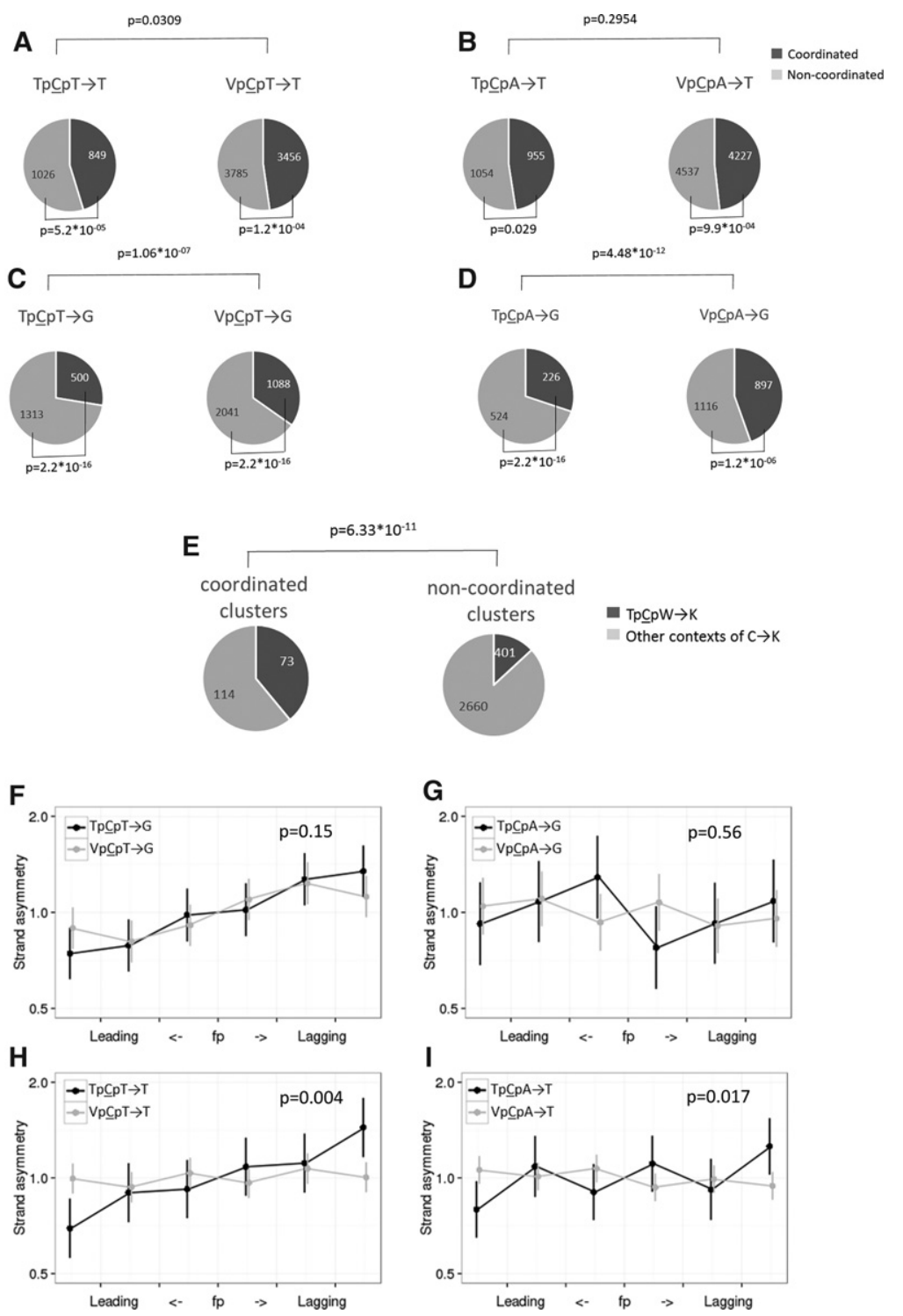

Figure 3. Clustered mutations in the $\mathrm{Tp} \mathrm{CpW} \rightarrow \mathrm{K}$ context are enriched in strand-coordinated clusters and biased toward the lagging strand. $(A-D)$ Pairs of linked SNPs in contexts prone to APOBEC $3 A / B$-induced mutations tend to be strand coordinated. (E) Strand-coordinated clusters that are composed exclusively of $\mathrm{C} \rightarrow \mathrm{K}$ mutations and contain at least six mutations are approximately threefold enriched in mutations in the TpCpW context, compared with noncoordinated clusters. (F-I) Strand-coordinated pairs are biased toward the lagging strand. The axes and notation are as in Figure 1. The $P$-value is cal-

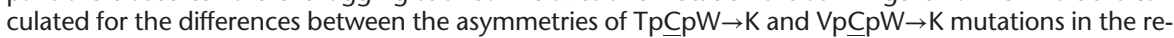
gions with the highest fork polarity (i.e., the first and the last bin).

\section{An unknown mechanism produces clusters of heritable $\mathrm{NpCpT} \rightarrow \mathrm{G}$ mutations}

Notably, a tendency to form strand-coordinated clusters was observed not only for the $\mathrm{Tp} \underline{\mathrm{p} W} \rightarrow \mathrm{K}$ mutations but also, to a lesser extent, for the $\mathrm{VpCpW} \rightarrow \mathrm{K}$ mutations (Fig. 3A-D). To better understand this, we focused on the VpCpT $\rightarrow$ G mutations for which this trend is particularly strong (Fig. 3C) and which are also biased toward the lagging strand (Fig. 1A). Notably, $C \rightarrow G$ is among the most frequent mutations in de novo mutational clusters (Francioli et al. 2015). We investigated $\mathrm{C} \rightarrow \mathrm{G}$ mutations in more detail in two recently published whole-genome data sets on human trios (Francioli et al. 2015; Wong et al. 2016) and found that within 23 pairs of $\mathrm{C} \rightarrow \mathrm{G}$ mutations at distances of up to $20 \mathrm{~kb}$ from each other, 18 were strand coordinated, which is more than expected if they were independent $(P=0.01)$ (Fig. 4A). The context preferences of the clustered $\mathrm{C} \rightarrow \mathrm{G}$ mutations differed from those of nonclustered mutations $(P=0.019)$, with the clustered $\mathrm{C} \rightarrow \mathrm{G}$ mutations enriched in the NpCpT $\rightarrow$ G context $(P=0.04)$ (Fig. 4C). Therefore, while the mechanism behind the strand-coordinated NpCpT $\rightarrow$ G mutations remains unclear, they are observed in all data types.

\section{Density of SNPs is increased at $3^{\prime}$ from $\mathrm{C} \rightarrow \mathrm{G}$ mutation}

In cancers, up to half of APOBEC3A/B-induced mutations are $\mathrm{C} \rightarrow \mathrm{G}$. These mutations arise due to bypass of abasic sites that originate from unfinished repair of cytosines deaminated by APOBEC3A/B. These and other DNA damages may be bypassed by low fidelity polymerase $\zeta$. It introduces $C>G$ mutations and is recruited to bypass different DNA damages (Diaz et al. 2003; Helleday et al. 2014) by replicating a stretch of DNA downstream

\section{Genome Research}

www.genome.org 
from the damage (Kochenova et al. 2015). If similar processes affect heritable mutations, we expect to observe a higher mutation rate at $3^{\prime}$ of the $\mathrm{C} \rightarrow \mathrm{G}$ mutations that mark polymerase $\zeta$-dependent DNA synthesis. We compared the mutation rates at distances

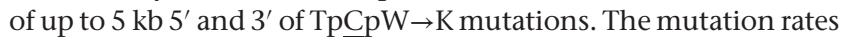
were increased $\sim 1-2 \mathrm{~kb} 3^{\prime}$ of $\mathrm{C} \rightarrow \mathrm{G}$ mutations in the APOBEC3A/B contexts both on the leading and the lagging strand (Fig. 5A,C, $\mathrm{E}, \mathrm{G})$. A higher mutation rate $3^{\prime}$ of $\mathrm{TpCpW} \rightarrow \mathrm{G}$ SNPs on both replicative strands at distances of up to $2 \mathrm{~kb}$ is in agreement with the key role of polymerase $\zeta$ in generation of these mutations and with its known low ( 1 kb) processivity (Kochenova et al. 2015). However, an increase in the mutation rate is also observed $3^{\prime}$ of $\mathrm{VpCpW} \rightarrow \mathrm{G}$ mutations (Supplemental Fig. S7) and on both chains in the case of APOBEC3A/B-prone context, implying that the role of polymerase $\zeta$ in the accumulation of heritable $\mathrm{C} \rightarrow \mathrm{G}$ mutations is not limited to bypassing of APOBEC3A/B-induced abasic sites.

A weaker effect was observed in one of the four comparisons for the $\mathrm{C} \rightarrow \mathrm{T}$ mutation (Fig. $5 \mathrm{~B}$ ): a mutation rate asymmetry in the vicinity of $\mathrm{Tp} \underline{\mathrm{CpT}} \rightarrow \mathrm{T}$ mutations on the lagging strand. This effect is particularly strong in the $400 \mathrm{nt}$ nearest to the $\mathrm{C} \rightarrow \mathrm{T}$ mutation (Supplemental Fig. S8). The TpCpT $\rightarrow \mathrm{T}$ mutations demonstrate the strongest bias toward the lagging strand compared with the control mutation types (Fig. 1), suggesting that APOBEC3A/B causes a high fraction of these mutations on the lagging strand. Therefore, our observations suggest that the mutation rate is increased $3^{\prime}$ of APOBEC $3 \mathrm{~A} / \mathrm{B}$-induced mutations. The difference between the mutation rates $5^{\prime}$ and $3^{\prime}$ of the mutation of interest is observed only for linked SNPs (Supplemental Fig. S9), implying that this association is due to the co-occurrence of mutations in a single mutational event rather than some underlying properties of the corresponding genomic regions.

The rate of all mutations is also strongly increased in the vicinity of a SNP in the same haplotype (Fig. 5), in line with previous results (Schrider et al. 2011; Terekhanova et al. 2013; Harris and Nielsen 2014; Zhu et al. 2015). This effect is weaker and decays more gradually with distance between the mutations not linked with each other compared with linked mutations (cf. Supplemental Fig. S9 and Fig. 5).

\section{Discussion}

Recent studies have linked most somatic mutations, that is, mutations associated with cancers or with dedifferentiation of induced pluripotent stem cells, with specific processes (Alexandrov et al. 2013; Lawrence et al. 2013; Rouhani et al. 2016). In contrast, only a minority of heritable mutations are attributable to known mechanisms. Analyses of clustered mutations and L1 transposons have revealed a major role of low fidelity polymerase $\zeta$ and APOBEC3G in the generation of heritable mutations (Harris and Nielsen 2014; Seplyarskiy et al. 2015; Zhu et al. 2015; Knisbacher and Levanon 2016; Pinto et al. 2016). Direct experiments have also uncovered the role of recombination in mutagenesis (Arbeithuber et al. 2015; Yang et al. 2015). Still, the causes of many described and pervasive patterns observed in heritable mutations such as the cryptic variation of the site-specific mutation rate and heterogeneity of mutational spectra (Hodgkinson et al. 2009; Johnson and Hellmann 2011; Seplyarskiy et al. 2012) remain unknown.

Among mutation types, clusters of adjacent or nearby mutations can be attributed to specific mutational mechanisms most reliably, as chance occurrence of such clusters by conventional mechanisms is unlikely. However, the majority of mutations are dispersed, and few methods to study their origin are available. A type of mutation can be relatively easily linked to a specific mechanism only if its rate is unusually high, as is the case for $\underline{\mathrm{CpG} \rightarrow \mathrm{T}}$ mutations.

Here, we show that APOBEC3A/B contributes substantially to heritable mutagenesis. We show that $\mathrm{Tp} \underline{\mathrm{CpW}} \rightarrow \mathrm{K}$ mutations in human polymorphism and divergence exhibit all the properties of APOBEC3A/B-induced mutations, unlike non-APOBECinduced $\mathrm{VpCpW} \rightarrow \mathrm{K}$ mutations. We estimate that $\sim 20 \%$ of heritable $\mathrm{Tp} \underline{\mathrm{pW}} \rightarrow \mathrm{K}$ mutations, or $1.4 \%$ of all heritable mutations, are linked to APOBEC3A/B activity, corresponding to 970,000 of mutations from the 1000 Genomes Project Consortium (2015).

Replication asymmetry of $\mathrm{TpCpW} \rightarrow \mathrm{K}$ mutations is not due to asymmetry in coreplicative repair or error rate of replicative polymerases

Many mutations are associated with replication and preferentially occur at segments of DNA strands replicated as leading or lagging. A computational approach has been developed to discriminate, by determining the prevalent direction of the replication fork at each genomic region, between the leading and the lagging DNA strands (Chen et al. 2011; Baker et al. 2012; Haradhvala et al. 2016; Seplyarskiy et al. 2016), providing a powerful tool to investigate strand-biased mechanisms that give rise even to dispersed mutations (Chen et al. 2011; Baker et al. 2012; Haradhvala et al. 2016; Morganella et al. 2016; Nik-Zainal et al. 2016; Seplyarskiy et al. 2016). APOBEC3A/B-induced mutations, as well as mutations in mismatch repair (MMR)-deficient cells, are strongly biased toward the lagging strand (Lujan et al. 2012; Andrianova et al. 2016;
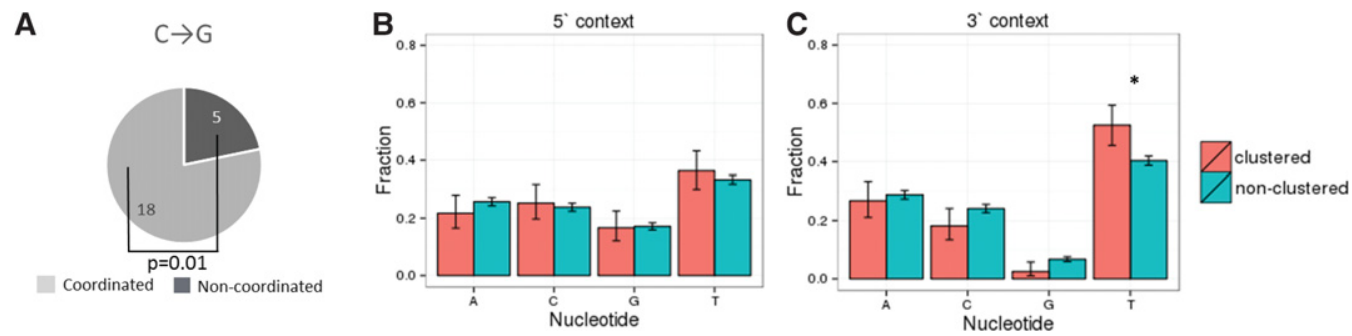

Figure 4. Properties of $C \rightarrow G$ mutations involved in clusters of de novo mutations. ( $A$ ) Pairs of $C \rightarrow G$ de novo mutations tend to form strand-coordinated clusters. $(B, C)$ Fractions of different nucleotides at adjacent sites $5^{\prime}(B)$ and $3^{\prime}(C)$ of the mutated position calculated for clustered and dispersed $C \rightarrow G$ de novo mutations. Vertical bars represent $95 \%$ confidence intervals. $\left(^{*}\right) P<0.05\left(\chi^{2}\right.$ test). 

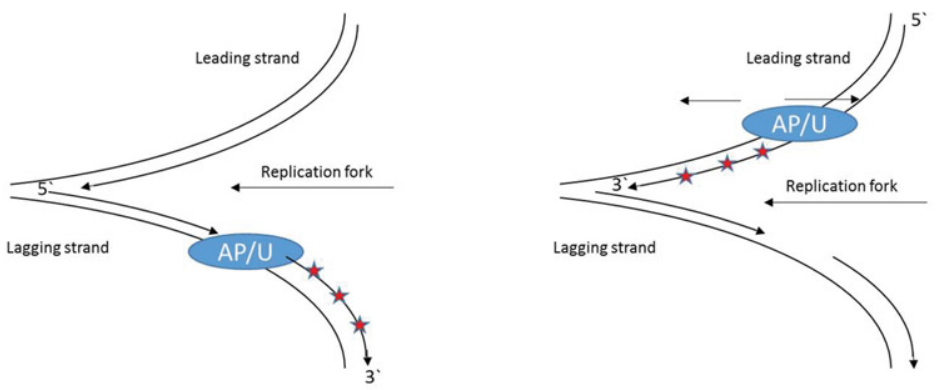

A

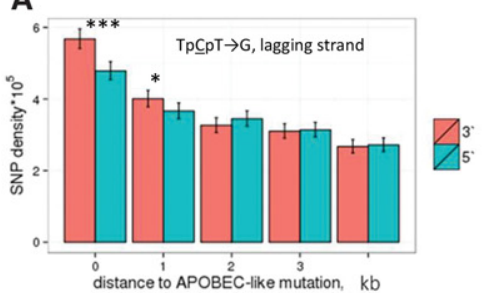

B

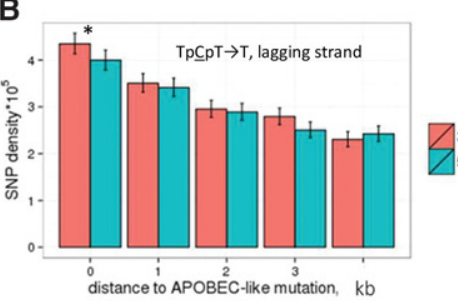

C

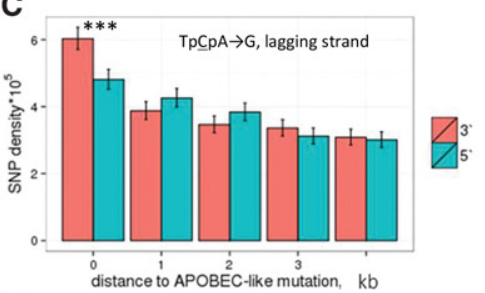

D

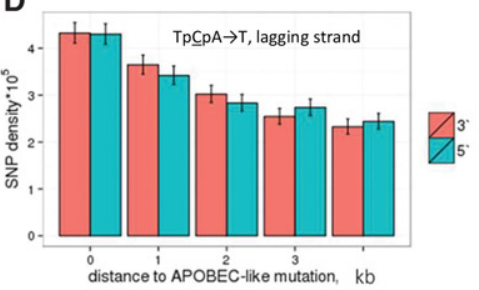

E

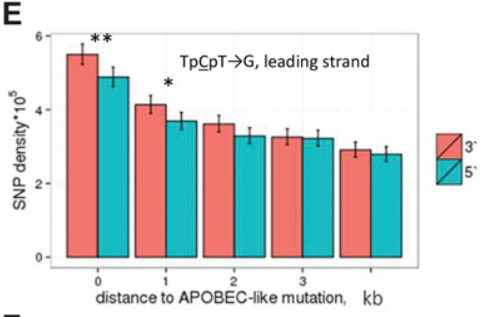

$\mathbf{F}$

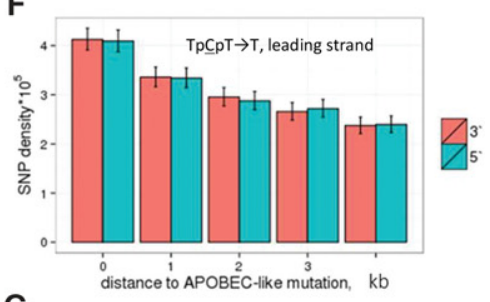

G

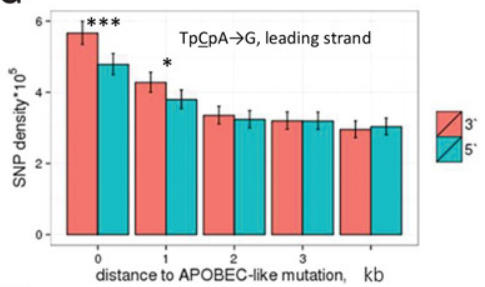

H

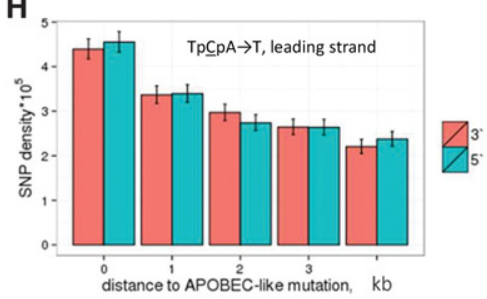

Figure 5. Density of linked SNPs $5^{\prime}$ and $3^{\prime}$ of a TpCpW $\rightarrow$ K mutation. In the schematic depiction of a replication fork at the top of the figure, red asterisks correspond to de novo mutations, and AP/U is the position of the $\mathrm{TpCpW} \rightarrow \mathrm{G}$ mutation interpreted as the position of the abasic site or the position of the TpCpW $\rightarrow$ T mutation interpreted as the position of the uracil. The rate of mutations on the lagging $(A-D)$ or the leading $(E-H)$ strand is measured in five nonoverlapping $1-\mathrm{kb}$ windows at increasing distance from the TpCpW $\rightarrow$ K SNPs. $\left({ }^{* * *}\right) P<0.001 ;\left({ }^{* *}\right) P<0.01 ;\left({ }^{*}\right) P<0.05$ ( $\chi^{2}$ test).

Haradhvala et al. 2016; Hoopes et al. 2016; Seplyarskiy et al. 2016). Here, we show that such biases in the germline shape the mutational lanscape that gives rise to human variation.

Conceivably, the observed replication asymmetry could reflect accumulation of nonrepaired mismatches during DNA doubling. Indeed, mutations accumulated in cells with a deficiency in MMR or decreased fidelity of major replicative leading or lagging strand polymerases have a strong replicative asymmetry (Lujan et al. 2012, 2014; Andrianova et al. 2016; Haradhvala et al. 2016). However, the direction of the observed replicative asymmetry is inconsistent with this. Indeed, in cancer cells prone to mutations caused by wild-type polymerases or by polymerase $\varepsilon$ or polymerase $\delta$ without exonuclease activity, $\mathrm{C} \rightarrow \mathrm{T}$ mutations are accumulated on the leading strand (Andrianova et al. 2016). In contrast, heritable $\mathrm{C} \rightarrow \mathrm{T}$ mutations in the $\mathrm{TpCpW}$ context preferentially occurred on the lagging strand (Fig. 1). Therefore, a specific context-dependent coreplicative mutational process needs to be invoked.

\section{APOBEC3A and/or APOBEC3B proteins are most plausible causes of the replicative asymmetry}

The asymmetry is most pronounced for cytosines in the TpCpW context, is associated with DNA replication, and produces strand-coordinated clusters, strongly suggesting that a protein from the APOBEC family plays a key role. While deamination in the TpCpW context excludes some APOBECs from consideration, APOBEC1, APOBEC3A, APOBEC $3 B, A P O B E C 3 F$, and АРОВEC $3 \mathrm{H}$ (Taylor et al. 2013; Kim et al. 2014; Saraconi et al. 2014) all are plausible suspects. Overexpression of APOBEC1, APOBEC $3 \mathrm{~A}$, and APOBEC3B is associated with increased mutation rate in vertebrate cell lines, with mutations distributed all over the genome (Saraconi et al. 2014; Akre et al. 2016; Green et al. 2016). Notably, however, APOBEC1 causes $\mathrm{C} \rightarrow \mathrm{A}$ mutations in experimental systems (Saraconi et al. 2014), while the heritable replication asymmetry is largely restricted to $\mathrm{C} \rightarrow \mathrm{T}$ and $\mathrm{C} \rightarrow \mathrm{G}$ mutations (Table 1). Recently, one of the alleles of APOBEC3H has been linked with lung cancer (Starrett et al. 2016), suggesting this protein as another possible candidate. However, this APOBEC3H allele is barely stable and has only a weak effect even in specific essays (Starrett et al. 2016), arguing against the role of APOBEC $3 \mathrm{H}$ in the patterns observed in germline. For APOBEC $3 \mathrm{~A}$ and APOBEC3B, mutations are known to accumulate on the lagging strand (Haradhvala et al. 2016; Hoopes et al. 2016; Seplyarskiy et al. 2016), and mutagenesis is associated with replication (Green et al. 2016). Finally, APOBEC $3 A$ and APOBEC $3 B$ are major mutators in a broad range of cancer types (Alexandrov et al. 2013; Burns et al. 2013b; Roberts et al. 2013). Therefore, although we cannot indisputably exclude other APOBECs, indirect evidence concordantly suggests $\mathrm{APOBEC} 3 \mathrm{~A}$ and $\mathrm{APOBEC} 3 \mathrm{~B}$ as the most probable

\section{Genome Research}

www.genome.org 
candidates. Our analysis of extended contexts make APOBEC3A a somewhat better explanation than APOBEC3B (Supplemental Fig. S2), although both proteins may contribute.

\section{Leading vs. lagging strand bias indicates that $20 \%$ of heritable} mutations are induced by APOBEC3A/B

For all types of heritable single-nucleotide substitutions, the asymmetry between the leading and the lagging strand is weak (Chen et al. 2011; Andrianova et al. 2016). Therefore, even a small admixture of mutations that are two to three times more prevalent on one of the strands can be detected (Fig. 1), and the amount of such admixture can be estimated from the level of asymmetry. De novo TpCpW $\rightarrow \mathrm{K}$ mutations obtained from Francioli et al. (2015) were inferred to be 1.38 times more frequent on the lagging strand than on the leading strand (Haradhvala et al. 2016). In our analyses of the same data set, the corresponding ratio is 1.23 but is not significantly different from $1.0(P>0.05)$. The difference between the results is likely due to differences in how $\mathrm{fp}$ is measured. The only assumption made by the method we use is that replication fork velocity is nearly constant throughout the genome (Guilbaud et al. 2011; Baker et al. 2012), and this assumption has been confirmed experimentally (Guilbaud et al. 2011; Baker et al. 2012). Still, the strand asymmetry of $\mathrm{Tp} \underline{\mathrm{CpW}} \rightarrow \mathrm{K}$ de novo mutations in a larger joined data set (Francioli et al. 2015; Wong et al. $2016)$ yielded a significant 1.17 -fold difference between strands $(P$ value $=0.0174$ ). This is in line with the approximately 1.15 -fold asymmetry observed for rare SNPs. The observed $\sim 15 \%$ asymmetry corresponds to a $\sim 20 \%$ admixture of APOBEC3A/B-induced mutations. Still, the effect of adjacent nucleotides on the mutation rate appears to mask this contribution of APOBEC $3 \mathrm{~A} / \mathrm{B}$, so that we do not observe any tendency of $\mathrm{C} \rightarrow \mathrm{K}$ mutations to occur in the TpCpW context among de novo mutations or in polymorphism data (Supplemental Tables S3, S7), in line with Francioli et al. (2015).

Our results show that knowledge of replication fork direction may be used to estimate the contribution of a specific process to heritable mutagenesis. We suggest that a similar set of approaches can be also used to quantitatively estimate the fraction of APOBEC3G-induced mutations, especially among dispersed mutations, because recent experiments on bacteria showed that these mutations, too, are strand asymmetric (Bhagwat et al. 2016).

\section{$\mathrm{Tp} \underline{\mathrm{CW}} \rightarrow \mathrm{K}$ mutations demonstrate transcriptional asymmetry}

Some mutation types are known to be transcriptionally asymmetric in human germline or soma (Green et al. 2003; Polak and Arndt 2008; Mugal et al. 2009; Pleasance et al. 2010; Nordentoft et al. 2014; Haradhvala et al. 2016). Here, we describe context-specific transcriptional asymmetry for $\mathrm{C} \rightarrow \mathrm{T}$ and $\mathrm{C} \rightarrow \mathrm{G}$ mutations in the germline (Fig. 2C). This asymmetry is elevated in TpCpW contexts. Transcription-coupled repair is the main source of transcriptional asymmetry in cancers (Pleasance et al. 2010; Haradhvala et al. 2016). However, if transcription-coupled repair was the main cause of the observed transcriptional asymmetry in the germline, we would not expect the mutation rate to be elevated at either of the strands compared with the intergenic regions. In contrast, we found that $\mathrm{Tp} \underline{\mathrm{CpW}} \rightarrow \mathrm{K}$ SNPs were more frequent on the nontemplate strand compared with intergenic regions (Fig. 2A). Genes tend to be located in regions of early RT (Farkash-Amar et al. 2008) and experience high levels of background selection (Mu et al. 2011); these factors could also reduce the number of SNPs but should not increase it, especially in a strand-specific man- ner. Therefore, a specific mutational mechanism needs to be invoked to explain the bias of $\mathrm{Tp} \underline{\mathrm{CpW}} \rightarrow \mathrm{K}$ mutations toward the nontemplate strand. A similar asymmetry in the same context has been described for APOBEC3A/B-induced mutations in breast and bladder cancer (Nordentoft et al. 2014; Morganella et al. 2016), suggesting that the transcriptional asymmetry likely reflects APOBEC3A/B-induced heritable mutations on the nontemplate strand. As APOBEC3A/B-induced mutagenesis affects singlestranded DNA, we suggest that, alongside the lagging strand during replication, it may also produce mutations on the nontemplate strand in transcribed regions, due to single strangeness of this strand during R loop formation (Skourti-Stathaki and Proudfoot 2014). The asymmetry observed in the $\mathrm{Cp} \underline{\mathrm{CpW}}$ context may be related to the APOBEC3G transcription-associated activity that has been shown recently (Pinto et al. 2016).

\section{Fraction of APOBEC3A/B-induced mutations is high in strand-coordinated clusters}

In cancer, APOBEC3A/B gives rise to mutational clusters spanning $\sim 10 \mathrm{~kb}$ (Nik-Zainal et al. 2012; Roberts et al. 2013). Linked SNPs, especially young ones, may be used to study such events, as mutational clusters observed in them have not yet been disrupted by recombination and are still detectible at distances of a few $\mathrm{kb}$ (Harris and Nielsen 2014). Clustered mutations in the TpCpW context are enriched in strand-coordinated clusters by a factor of up to two. Moreover, for strand-coordinated clusters, we observed a stronger bias toward the lagging strand than for dispersed mutations. Thus, the orthogonal approach based on strand coordination also detects the prevalence of APOBEC3A/B-induced mutations.

\section{Contribution of APOBEC3A/B-induced mutations to the mutation load in humans}

APOBEC3A/B-induced mutations fuel cancer development, representing the second most prevalent mutational signature in it. Therefore, the cancer-related activity of APOBEC3A/B should be slightly deleterious, although selection against it may be weak (Martincorena and Campbell 2015). Here, we have shown that APOBEC $3 \mathrm{~A} / \mathrm{B}$ also causes heritable mutations, thus increasing the mutation load. The fact that it is conserved by negative selection implies that its positive role in protection against retroelements or viruses outweighs its deleterious mutability. Thus, the maintenance of APOBEC3A/B represents a tradeoff between its advantageous function and deleterious mutagenesis.

\section{Methods}

\section{Mutational data}

The main set of results was obtained using polymorphism data from the 1000 Genomes Project Consortium (2015). We excluded exons and $10 \mathrm{nt}$ adjacent to each exon to reduce the contribution of selection, and we only considered nonsingleton variants with low $(<1 \%)$ derived allele frequency, using the ancestral variant determined by the 1000 Genomes Project Consortium (2015). Clusters were defined as pair or more of SNPs of a particular type at distances between 10 and $5000 \mathrm{nt}$ from each other, such that at least half of the genotypes carrying the derived allele for one of the SNPs also carry the derived allele for any other SNP within cluster and vice versa. The same criteria were used to subdivide SNPs as linked (Fig. 5; Supplemental Figs. S8, S9) or unlinked (Supplemental Fig. S7) in analyses of SNP densities $5^{\prime}$ or $3^{\prime}$ of the considered SNP. For analyses of interspecies divergence, we used 
the human-chimpanzee-orangutan multiple alignment from the UCSC Genome Browser (https://genome.ucsc.edu/). We inferred substitutions in the human lineage after its divergence from the chimpanzee by maximum parsimony, using the orangu$\tan$ as the outgroup. Somatic mutations in cancers for wholegenome sequences were obtained from Alexandrov et al. (2013) and the TCGA consortium (Hoadley et al. 2014; https:// tcga-data.nci.nih.gov/tcga/). Mutational clusters in cancers were determined as described by Seplyarskiy et al. (2016) and mutational clusters of de novo heritable mutations obtained from trio data as described by Francioli et al. (2015). Cancers with a strong prevalence of APOBEC3A/B signature were defined on the basis of an increased rate of the $\mathrm{TpCpW} \rightarrow \mathrm{K}$ mutation; this matches the definition of APOrich cancers in the work by Seplyarskiy et al. (2016). Mutation rates in all analyses were calculated as the number of events divided on the number of corresponding sites.

We have written Perl scripts (Supplemental perl scripts) and R scripts (Supplemental R scripts) to analyze the data.

\section{Genomic annotations}

Information about genomic coordinates and which strand is the template for transcribed genes and expressed transposons was downloaded from the UCSC Genome Browser, files knownGene and ucscRetroAli5, correspondingly. Hotspots of meiotic doublestrand breaks, also called recombination hotspots, were obtained from Pratto et al. (2014). We use all hotspots observed in at least one individual (file 1256442_DatafileS1.txt, column 17).

\section{Leading vs. lagging strand asymmetry}

The derivative of the replication timing at the position of the mutation was used as a proxy for the probability that the reference strand is replicated as leading or lagging in the current position, as previously described (Seplyarskiy et al. 2016). The genome was categorized by these values into six (Fig. 3F-I) or 20 (Fig. 1) equal bins, with low value of the derivative corresponding to the propensity of the DNA segment to be replicated as lagging; high value, as leading. For each bin, the numbers of substitutions and target sites were calculated. Each substitution was counted twice: (1) as a substitution on the reference strand with the corresponding derivative of the replication timing and (2) as a complementary substitution with the inverse derivative. Thus, each plot of substitution asymmetry (Figs. 1, 3F-I) is symmetric with respect to zero. Confidence intervals were obtained for the relative risk in a $2 \times 2$ table. As a measure of the asymmetry, we used the ratio of the frequencies of complementary mutations between strands in the most extreme (first or last) bin, where the determination of the fp was the most confident. Since tissue-matched replication timing for heritable mutations is unavailable, MCF-7 replication timing was used for the main analysis. Results replicated for all seven cell types with available RT data (Supplemental data fp). To reshuffle MCF-7 RTs, we randomly picked a value of RT for each $1-\mathrm{kb}$ segment from the distribution of MCF-7 RT values.

\section{Estimation of the admixture of APOBEC3A/B mutations from the leading vs. lagging strand asymmetry}

Previously, using non-tissue-specific replication timing data as in the current analysis of heritable mutations, we found that about two-thirds of APOBEC3A/B mutations in cancer occurred on the lagging strand (Seplyarskiy et al. 2016). By using tissue-matched data on replication timing for analyses of asymmetry in cancers (MCF-7 for breast cancers, and IMR90 for lung cancers), we increase the observed level of asymmetry (Supplemental Fig. S10) compared with our previous study and studies by other groups
(Haradhvala et al. 2016; Morganella et al. 2016; Seplyarskiy et al. 2016). Therefore, in our non-tissue-matched data, the observed ratio of mutational frequencies on the lagging and on the leading strands (strand bias) $s$ is

$$
s=\frac{0.67 x+0.5(1-x)}{0.33 x+0.5(1-x)},
$$

where $x$ is the fraction of APOBEC3A/B-induced mutations among all mutations; $0.67 x$ and $0.33 x$ are the fractions of APOBEC $3 \mathrm{~A} / \mathrm{B}-$ induced mutations on the lagging and on the leading strand, respectively, with the coefficients 0.67 and 0.33 estimated from cancer data; and the coefficient 0.5 corresponds to the equal distribution of non-APOBEC3A/B-induced mutations between leading and lagging strands. Therefore,

$$
x=\frac{3(s-1)}{1+s},
$$

so that $s=1.15$ estimated for heritable mutations implies an admixture of APOBEC3A/B-induced mutations of $21 \%$.

\section{Acknowledgments}

We thank Wendy Wong, Shamil Sunyaev, Ruslan Soldatov, and Nadezhda Terekhanova for useful discussion and Wendy Wong for help with data retrieving from the study by Wong et al. (2016). This work was performed in IITP RAS and supported by the Russian Science Foundation (grant no. 14-50-00150).

Author contributions: V.B.S. conceived the study and performed the analyses; all authors contributed to study design, intensively discussed the study, and wrote the manuscript.

\section{References}

The 1000 Genomes Project Consortium. 2015. A global reference for human genetic variation. Nature 526: 68-74.

Aggarwala V, Voight BF. 2016. An expanded sequence context model broadly explains variability in polymorphism levels across the human genome. Nat Genet 48: 349-355.

Akre MK, Starrett GJ, Quist JS, Temiz NA, Carpenter MA, Tutt ANJ, Grigoriadis A, Harris RS. 2016. Mutation processes in 293-based clones overexpressing the DNA cytosine deaminase APOBEC3B. PLoS One 11: e0155391.

Alexandrov LB, Nik-Zainal S, Wedge DC, Aparicio SAJR, Behjati S, Biankin AV, Bignell GR, Bolli N, Borg A, Børresen-Dale A-L, et al. 2013. Signatures of mutational processes in human cancer. Nature 500: $415-421$.

Andrianova M, Bazykin GA, Nikolaev S, Seplyarskiy V. 2016. Human mismatch repair system corrects errors produced during lagging strand replication more effectively. bioRxiv 45278. doi: https://doi.org/10.1101/ 045278 .

Arbeithuber B, Betancourt AJ, Ebner T, Tiemann-Boege I. 2015. Crossovers are associated with mutation and biased gene conversion at recombination hotspots. Proc Natl Acad Sci 112: 2109-2114.

Baker A, Audit B, Chen C-L, Moindrot B, Leleu A, Guilbaud G, Rappailles A, Vaillant C, Goldar A, Mongelard F, et al. 2012. Replication fork polarity gradients revealed by megabase-sized U-shaped replication timing domains in human cell lines. PLoS Comput Biol 8: e1002443.

Bhagwat AS, Hao W, Townes JP, Lee H, Tang H, Foster PL. 2016. Strand-biased cytosine deamination at the replication fork causes cytosine to thymine mutations in Escherichia coli. Proc Natl Acad Sci 113: 2176-2181.

Burns MB, Lackey L, Carpenter MA, Rathore A, Land AM, Leonard B Refsland EW, Kotandeniya D, Tretyakova N, Nikas JB, et al. 2013a. APOBEC3B is an enzymatic source of mutation in breast cancer Nature 494: 366-370.

Burns MB, Temiz NA, Harris RS. 2013b. Evidence for APOBEC3B mutagenesis in multiple human cancers. Nat Genet 45: 977-983.

Chan K, Resnick MA, Gordenin DA. 2013. The choice of nucleotide inserted opposite abasic sites formed within chromosomal DNA reveals the polymerase activities participating in translesion DNA synthesis. DNA Repair 12: $878-889$.

Chan K, Roberts SA, Klimczak LJ, Sterling JF, Saini N, Malc EP, Kim J, Kwiatkowski DJ, Fargo DC, Mieczkowski PA, et al. 2015. An 
APOBEC3A hypermutation signature is distinguishable from the signature of background mutagenesis by APOBEC3B in human cancers. Nat Genet 47: 1067-1072.

Chen C-L, Duquenne L, Audit B, Guilbaud G, Rappailles A, Baker A, Huvet M, d'Aubenton-Carafa Y, Hyrien O, Arneodo A, et al. 2011. Replicationassociated mutational asymmetry in the human genome. Mol Biol Evol 28: 2327-2337.

Chen J, Miller BF, Furano AV. 2014. Repair of naturally occurring mismatches can induce mutations in flanking DNA. eLife 3: e02001.

Diaz M, Watson NB, Turkington G, Verkoczy LK, Klinman NR, McGregor WG. 2003. Decreased frequency and highly aberrant spectrum of ultraviolet-induced mutations in the hprt gene of mouse fibroblasts expressing antisense RNA to DNA polymerase $\zeta$. Mol Cancer Res 1: 836-847.

Farkash-Amar S, Lipson D, Polten A, Goren A, Helmstetter C, Yakhini Z, Simon I. 2008. Global organization of replication time zones of the mouse genome. Genome Res 18: 1562-1570.

Francioli LC, Polak PP, Koren A, Menelaou A, Chun S, Renkens I, Genome of the Netherlands Consortium, van Duijn CM, Swertz M, Wijmenga C, et al. 2015. Genome-wide patterns and properties of de novo mutations in humans. Nat Genet 47: 822-826.

Goldmann JM, Wong WSW, Pinelli M, Farrah T, Bodian D, Stittrich AB, Glusman G, Vissers LELM, Hoischen A, Roach JC, et al. 2016. Parentof-origin-specific signatures of de novo mutations. Nat Genet 48: 935-939.

Green P, Ewing B, Miller W, Thomas PJ, NISC Comparative Sequencing Program, Green ED. 2003. Transcription-associated mutational asymmetry in mammalian evolution. Nat Genet 33: 514-517.

Green AM, Landry S, Budagyan K, Avgousti DC, Shalhout S, Bhagwat AS, Weitzman MD. 2016. APOBEC3A damages the cellular genome during DNA replication. Cell Cycle 15: $998-1008$.

Guilbaud G, Rappailles A, Baker A, Chen C-L, Arneodo A, Goldar A, d'Aubenton-Carafa Y, Thermes C, Audit B, Hyrien O. 2011. Evidence for sequential and increasing activation of replication origins along replication timing gradients in the human genome. PLoS Comput Biol 7: e1002322.

Haradhvala NJ, Polak P, Stojanov P, Covington KR, Shinbrot E, Hess JM, Rheinbay E, Kim J, Maruvka YE, Braunstein LZ, et al. 2016. Mutational strand asymmetries in cancer genomes reveal mechanisms of DNA damage and repair. Cell 164: 538-549.

Harris K, Nielsen R. 2014. Error-prone polymerase activity causes multinucleotide mutations in humans. Genome Res 24: 1445-1454.

Helleday T, Eshtad S, Nik-Zainal S. 2014. Mechanisms underlying mutational signatures in human cancers. Nat Rev Genet 15: 585-598.

Hoadley KA, Yau C, Wolf DM, Cherniack AD, Tamborero D, Ng S, Leiserson MDM, Niu B, McLellan MD, Uzunangelov V, et al. 2014. Multiplatform analysis of 12 cancer types reveals molecular classification within and across tissues of origin. Cell 158: 929-944.

Hodgkinson A, Ladoukakis E, Eyre-Walker A. 2009. Cryptic variation in the human mutation rate. PLoS Biol 7: e1000027.

Hoopes JI, Cortez LM, Mertz TM, Malc EP, Mieczkowski PA, Roberts SA. 2016. APOBEC3A and APOBEC3B preferentially deaminate the lagging strand template during DNA replication. Cell Rep 14: 1273-1282.

Johnson PLF, Hellmann I. 2011. Mutation rate distribution inferred from coincident SNPs and coincident substitutions. Genome Biol Evol 3: 842-850.

Kim E-Y, Lorenzo-Redondo R, Little SJ, Chung Y-S, Phalora PK, Maljkovic Berry I, Archer J, Penugonda S, Fischer W, Richman DD, et al. 2014. Human APOBEC3 induced mutation of human immunodeficiency virus type- 1 contributes to adaptation and evolution in natural infection. PLoS Pathog 10: e1004281.

Knisbacher BA, Levanon EY. 2016. DNA editing of LTR retrotransposons reveals the impact of APOBECs on vertebrate genomes. Mol Biol Evol 33: 554-567.

Kochenova OV, Daee DL, Mertz TM, Shcherbakova PV. 2015. DNA polymerase $\zeta$-dependent lesion bypass in Saccharomyces cerevisiae is accompanied by error-prone copying of long stretches of adjacent DNA. PLoS Genet 11: e1005110.

Kong A, Frigge ML, Masson G, Besenbacher S, Sulem P, Magnusson G, Gudjonsson SA, Sigurdsson A, Jonasdottir A, Jonasdottir A, et al. 2012. Rate of de novo mutations and the importance of father's age to disease risk. Nature 488: 471-475.

Koren A, Handsaker RE, Kamitaki N, Karlić R, Ghosh S, Polak P, Eggan K, McCarroll SA. 2014. Genetic variation in human DNA replication timing. Cell 159: 1015-1026.

Lawrence MS, Stojanov P, Polak P, Kryukov GV, Cibulskis K, Sivachenko A, Carter SL, Stewart C, Mermel CH, Roberts SA, et al. 2013. Mutational heterogeneity in cancer and the search for new cancer-associated genes. Nature 499: 214-218.
Lujan SA, Williams JS, Pursell ZF, Abdulovic-Cui AA, Clark AB, Nick McElhinny SA, Kunkel TA. 2012. Mismatch repair balances leading and lagging strand DNA replication fidelity. PLoS Genet 8: e1003016.

Lujan SA, Clausen AR, Clark AB, MacAlpine HK, MacAlpine DM, Malc EP, Mieczkowski PA, Burkholder AB, Fargo DC, Gordenin DA, et al. 2014. Heterogeneous polymerase fidelity and mismatch repair bias genome variation and composition. Genome Res 24: 1751-1764.

Martincorena I, Campbell PJ. 2015. Somatic mutation in cancer and normal cells. Science 349: 1483-1489.

Mathieson I, McVean G. 2014. Demography and the age of rare variants. PLoS Genet 10: e1004528.

Mathieson I, Reich DE. 2016. Variation in mutation rates among human populations. bioRxiv 63578. doi: https://doi.org/10.1101/063578.

Montgomery SB, Goode DL, Kvikstad E, Albers CA, Zhang ZD, Mu XJ, Ananda G, Howie B, Karczewski KJ, Smith KS, et al. 2013. The origin, evolution, and functional impact of short insertion-deletion variants identified in 179 human genomes. Genome Res 23: 749-761.

Morganella S, Alexandrov LB, Glodzik D, Zou X, Davies H, Staaf J, Sieuwerts AM, Brinkman AB, Martin S, Ramakrishna M, et al. 2016. The topography of mutational processes in breast cancer genomes. Nat Commun 7: 11383.

Mu XJ, Lu ZJ, Kong Y, Lam HYK, Gerstein MB. 2011. Analysis of genomic variation in non-coding elements using population-scale sequencing data from the 1000 Genomes Project. Nucleic Acids Res 39: 7058-7076.

Mugal CF, von Grünberg H-H, Peifer M. 2009. Transcription-induced mutational strand bias and its effect on substitution rates in human genes. Mol Biol Evol 26: 131-142.

Nik-Zainal S, Alexandrov LB, Wedge DC, Van Loo P, Greenman CD, Raine K, Jones D, Hinton J, Marshall J, Stebbings LA, et al. 2012. Mutational processes molding the genomes of 21 breast cancers. Cell 149: 979-993.

Nik-Zainal S, Davies H, Staaf J, Ramakrishna M, Glodzik D, Zou X, Martincorena I, Alexandrov LB, Martin S, Wedge DC, et al. 2016. Landscape of somatic mutations in 560 breast cancer whole-genome sequences. Nature 534: 47-54.

Nordentoft I, Lamy P, Birkenkamp-Demtröder K, Shumansky K, Vang S, Hornshøj H, Juul M, Villesen P, Hedegaard J, Roth A, et al. 2014 Mutational context and diverse clonal development in early and late bladder cancer. Cell Rep 7: 1649-1663.

Pfeifer GP. 2006. Mutagenesis at methylated CpG sequences. Curr Top Microbiol Immunol 301: 259-281.

Pinto Y, Gabay O, Arbiza L, Sams AJ, Keinan A, Levanon EY. 2016. Clustered mutations in hominid genome evolution are consistent with APOBEC3G enzymatic activity. Genome Res 26: 579-587.

Pleasance ED, Cheetham RK, Stephens PJ, McBride DJ, Humphray SJ, Greenman CD, Varela I, Lin M-L, Ordóñez GR, Bignell GR, et al. 2010. A comprehensive catalogue of somatic mutations from a human cancer genome. Nature 463: 191-196.

Polak P, Arndt PF. 2008. Transcription induces strand-specific mutations at the $5^{\prime}$ end of human genes. Genome Res 18: 1216-1223.

Pratto F, Brick K, Khil P, Smagulova F, Petukhova GV, Camerini-Otero RD. 2014. DNA recombination. Recombination initiation maps of individual human genomes. Science 346: 1256442.

Rahbari R, Wuster A, Lindsay SJ, Hardwick RJ, Alexandrov LB, Al Turki S, Dominiczak A, Morris A, Porteous D, Smith B, et al. 2016. Timing, rates and spectra of human germline mutation. Nat Genet 48: 126-133.

Roberts SA, Lawrence MS, Klimczak LJ, Grimm SA, Fargo D, Stojanov P, Kiezun A, Kryukov GV, Carter SL, Saksena G, et al. 2013. An APOBEC cytidine deaminase mutagenesis pattern is widespread in human cancers. Nat Genet 45: 970-976.

Rouhani FJ, Nik-Zainal S, Wuster A, Li Y, Conte N, Koike-Yusa H, Kumasaka N, Vallier L, Yusa K, Bradley A. 2016. Mutational history of a human cell lineage from somatic to induced pluripotent stem cells. PLoS Genet 12: e1005932.

Saraconi G, Severi F, Sala C, Mattiuz G, Conticello SG. 2014. The RNA editing enzyme APOBEC1 induces somatic mutations and a compatible mutational signature is present in esophageal adenocarcinomas. Genome Biol 15: 417.

Schrider DR, Hourmozdi JN, Hahn MW. 2011. Pervasive multinucleotide mutational events in eukaryotes. Curr Biol 21: 1051-1054.

Seplyarskiy VB, Kharchenko P, Kondrashov AS, Bazykin GA. 2012. Heterogeneity of the transition/transversion ratio in Drosophila and Hominidae genomes. Mol Biol Evol 29: 1943-1955.

Seplyarskiy VB, Bazykin GA, Soldatov RA. 2015. Polymerase $\zeta$ activity is linked to replication timing in humans: evidence from mutational signatures. Mol Biol Evol 32: 3158-3172.

Seplyarskiy VB, Soldatov RA, Popadin KY, Antonarakis SE, Bazykin GA, Nikolaev SI. 2016. APOBEC-induced mutations in human cancers are strongly enriched on the lagging DNA strand during replication. Genome Res 26: 174-182. 
Shendure J, Akey JM. 2015. The origins, determinants, and consequences of human mutations. Science 349: 1478-1483.

Skourti-Stathaki K, Proudfoot NJ. 2014. A double-edged sword: R loops as threats to genome integrity and powerful regulators of gene expression. Genes Dev 28: 1384-1396.

Starrett GJ, Luengas EM, McCann JL, Ebrahimi D, Temiz NA, Love RP, Feng Y, Adolph MB, Chelico L, Law EK, et al. 2016. The DNA cytosine deaminase APOBEC3H haplotype I likely contributes to breast and lung cancer mutagenesis. Nat Commun 7: 12918.

Taylor BJ, Nik-Zainal S, Wu YL, Stebbings LA, Raine K, Campbell PJ, Rada C, Stratton MR, Neuberger MS. 2013. DNA deaminases induce break-associated mutation showers with implication of $A P O B E C 3 B$ and $3 \mathrm{~A}$ in breast cancer kataegis. eLife 2: e00534.

Terekhanova NV, Bazykin GA, Neverov A, Kondrashov AS, Seplyarskiy VB. 2013. Prevalence of multinucleotide replacements in evolution of primates and Drosophila. Mol Biol Evol 30: 1315-1325.

Terekhanova NV, Seplyarskiy V, Soldatov RA, Bazykin GA. 2016. Evolution of local mutation rate and its determinants. bioRxiv 54825. doi: https ://doi.org/10.1101/054825.
Wong WSW, Solomon BD, Bodian DL, Kothiyal P, Eley G, Huddleston KC, Baker R, Thach DC, Iyer RK, Vockley JG, et al. 2016. New observations on maternal age effect on germline de novo mutations. Nat Commun 7: 10486.

Yang S, Wang L, Huang J, Zhang X, Yuan Y, Chen J-Q, Hurst LD, Tian D. 2015. Parent-progeny sequencing indicates higher mutation rates in heterozygotes. Nature 523: 463-467.

Yuen RK, Merico D, Cao H, Pellecchia G, Alipanahi B, Thiruvahindrapuram B, Tong X, Sun Y, Cao D, Zhang T, et al. 2016. Genome-wide characteristics of de novo mutations in autism. NPJ Genom Med 1: 16027.

Zhu W, Cooper DN, Zhao Q, Wang Y, Liu R, Li Q, Férec C, Wang Y, Chen JM. 2015. Concurrent nucleotide substitution mutations in the human genome are characterized by a significantly decreased transition/transversion ratio. Hum Mutat 36: 333-341.

Received May 24, 2016; accepted in revised form December 1, 2016.

\section{Genome Research}




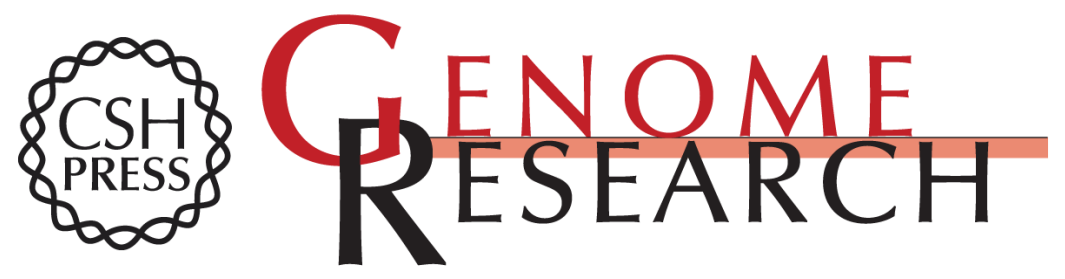

\section{APOBEC3A/B-induced mutagenesis is responsible for $20 \%$ of heritable mutations in the TpCpW context}

Vladimir B. Seplyarskiy, Maria A. Andrianova and Georgii A. Bazykin

Genome Res. 2017 27: 175-184 originally published online December 9, 2016

Access the most recent version at doi:10.1101/gr.210336.116

Supplemental Material

References

Creative

Commons

License

Email Alerting

Service
http://genome.cshlp.org/content/suppl/2017/01/13/gr.210336.116.DC1

This article cites 66 articles, 14 of which can be accessed free at: http://genome.cshlp.org/content/27/2/175.full.html\#ref-list-1

This article is distributed exclusively by Cold Spring Harbor Laboratory Press for the first six months after the full-issue publication date (see

$\mathrm{http}: / /$ genome.cshlp.org/site/misc/terms.xhtml). After six months, it is available under a Creative Commons License (Attribution-NonCommercial 4.0 International), as described at http://creativecommons.org/licenses/by-nc/4.0/.

Receive free email alerts when new articles cite this article - sign up in the box at the top right corner of the article or click here.

\section{Affordable, Accurate Sequencing.}

